\title{
Does suicidal desire moderate the association between frontal delta power and psychological pain?
}

Esther L. Meerwijk, Sandra J. Weiss

Psychological pain frequently underlies thoughts of suicide. We investigated if recent suicidal desire moderated the association between potential neurophysiological markers and psychological pain assessed on the Psychache Scale (PS) and the Orbach \& Mikulincer Mental Pain Questionnaire (OMMP). The OMMP specifically assesses current psychological pain that may more readily capture emotions present during recent suicidal desire. In contrast, the PS leaves the timeframe undefined. A secondary analysis was conducted of resting-state EEG data and heart rate obtained in adults with a history of depression. In simultaneous multiple regression models while controlling for depressive symptoms, recent suicidal desire moderated associations with right-frontal EEG delta power $\left(\Delta R^{2}=\right.$ $.07, p<.01$ ) and low-frequency heart rate variability (nonsignificantly) for pain assessed on the PS. No indication for moderation was found for pain on the OMMP. The relationship between the two measures of psychological pain was stronger for individuals with recent suicidal desire $(r=.75, p<.01$ vs. $r=.50, p<.05)$. The findings suggest that, unless a respondent's psychological pain is recent and substantial, the PS may not capture the intensity of current psychological pain as effectively as the OMMP. 
Does recent suicidal desire moderate the association between frontal delta power and psychological pain?

\author{
Esther L. Meerwijk* ${ }^{*}$ PhD \& Sandra J. Weiss, RN, PhD, DNSc
}

Department of Community Health Systems

University of California, San Francisco, CA, USA

\section{Abstract}

Psychological pain frequently underlies thoughts of suicide. We investigated if recent suicidal desire moderated the association between potential neurophysiological markers and psychological pain assessed on the Psychache Scale (PS) and the Orbach \& Mikulincer Mental Pain Questionnaire (OMMP). The OMMP specifically assesses current psychological pain that may more readily capture emotions present during recent suicidal desire. In contrast, the PS leaves the timeframe undefined. A secondary analysis was conducted of resting-state EEG data and heart rate obtained in adults with a history of depression. In simultaneous multiple regression models, while controlling for depressive symptoms, recent suicidal desire moderated associations with right-frontal EEG delta power $\left(\Delta R^{2}=.07, p<.01\right)$ and low-frequency heart rate variability (nonsignificantly) for pain assessed on the PS. No indication for moderation was found for pain on the OMMP. The relationship between the two measures of psychological pain was stronger for individuals with recent suicidal desire $(r=.75, p<.01$ vs. $r=.50, p<.05)$. The findings suggest that, unless a respondent's psychological pain is recent and substantial, the PS may not capture the intensity of current psychological pain as effectively as the OMMP.

\section{${ }^{*}$ Corresponding author contact information:}

Esther L. Meerwijk, Department of Community Health Systems, School of Nursing, University of California, San Francisco, 2 Koret Way, Suite N505, San Francisco, CA 941430608, (650) 391-4449, email: esther.meerwijk@gmail.com, Esther Meerwijk is currently a postdoctoral fellow with the UCSF Symptom Management Program. 


\section{Introduction}

Psychological pain is a subjective experience that involves negative appraisal of self, and as such requires self-report instruments to be assessed (Meerwijk \& Weiss, 2011; Tossani, 2013). Among the first self-report instruments of psychological pain were Shneidman's Psychological Pain Assessment Scale (Shneidman, 1999) and the Psychache Scale ([PS], Holden et al., 2001). These were followed by the Orbach \& Mikulincer Mental Pain Questionnaire ([OMMP], Orbach et al., 2003a) and more recently the Mee-Bunney Psychological Pain Assessment Scale (Mee et al., 2011) and the Three-dimensional Psychological Pain Scale (Li et al., 2014). Of these instruments, the PS and OMMP are probably the most frequently used to assess psychological pain.

While psychological pain is not unique to people with depression, ample research has found that people with depression report high levels of psychological pain and that hopelessness covaries with psychological pain (van Heeringen et al., 2010; Mee et al., 2011; Li et al., 2014; Xie et al., 2014; Cáceda et al., 2014; Troister, D’Agata \& Holden, 2015). We recently reported that psychological pain as assessed on the OMMP, but not the PS, in adults with a history of depression correlated with objective measures of resting-state neurophysiological parameters, in particular low-frequency heart rate variability (HRV) and right frontal EEG delta power (Meerwijk, Chesla \& Weiss, 2014; Meerwijk, Ford \& Weiss, 2015). As discussed in our previous publications, the relationship between psychological pain and these neurophysiological parameters may result from resting-state dysfunction of the sympathetic nervous system and increased sympathetic activity under conditions of greater psychological pain. Different findings when using these two psychological pain scales were unexpected because they purport to measure the same construct of psychological pain. In light of the association of neurophysiological markers with suicide ideation (Iosifescu et al., 2008; Hunter et al., 2010; Song et al., 2011; Chang et al., 2012), and the associations found between suicide ideation and psychological pain in depression (Olié et al., 2010; van Heeringen et al., 2010), we questioned whether recent suicidal desire of respondents could moderate and thus explain the differential findings obtained with the PS and OMMP in adults with a history of depression. The OMMP specifically assesses current psychological pain that may more readily capture emotions present during recent suicidal desire. In contrast, the PS leaves the timeframe undefined, allowing respondents to consider their lifetime experience when assessing psychological pain. However, individuals with recent suicidal desire would likely assess their pain on the PS in light of their current feelings. For these individuals, we would expect the relationship between the OMMP and PS scores to be stronger. In addition, we would expect their PS scores to be more strongly associated with resting-state neurophysiological parameters. We present here a secondary analysis that tested the following hypotheses: (1) the correlation between OMMP and PS scores 
71 will be stronger among individuals who report a recent desire for suicide than among individuals who do not report recent suicidal desire, and (2) the correlation of the PS score with lowfrequency heart rate variability and right frontal delta power in the resting state will be stronger among individuals who report a recent suicidal desire than among those who do not report recent suicidal desire.

Ample empirical evidence supports the notion that psychological pain is associated with suicidal thoughts and behaviors. Several studies observed a relationship between a history of suicide attempts and psychological pain, and found that psychological pain significantly predicted suicide attempter status (one or more vs. zero attempts), after controlling for depression and hopelessness (Pereira et al., 2010; Troister \& Holden, 2010; Patterson \& Holden, 2012). Significantly higher psychological pain was found in people who had a history of suicide attempts versus people who never attempted suicide (Orbach et al., 2003b; Flamenbaum \& Holden, 2007; Levi et al., 2008), but no significant differences were also reported (Pompili et al., 2008). Delisle and Holden (2009) found that psychological pain predicted reasons for attempting suicide, after controlling for depression and hopelessness, and Olié et al. (2010) found that patients who were hospitalized for a depressive episode and who had attempted suicide, reported high current psychological pain more often than inpatients who had not attempted suicide. While recent adverse events may trigger psychological pain and precipitate a suicide attempt, research has also shown that adverse events during early life can predispose an individual to suicide attempts during adulthood (Pompili et al., 2011). Levi et al. (2008) did not find a significant effect of psychological pain in inpatients with regard to the seriousness of suicide attempts.

Other studies reported on the relationship between the broader concept of suicide ideation and psychological pain. Medium to strong positive correlations were found between suicide ideation and psychological pain in undergraduate students (Lester, 2000; Holden et al., 2001; Leenaars \& Lester, 2004) and in patients admitted for a depressive episode (van Heeringen et al., 2010). In the only prospective studies of psychological pain to date (Troister \& Holden, 2012; Troister et al., 2013), change in psychological pain was the only predictor of suicide ideation over two years for undergraduate students who were thought to be at high risk of suicide at baseline. In a different study of undergraduates, Troister and Holden (2013) also reported that psychological pain was the stronger predictor of suicide ideation, partially mediating the contributions of depression and hopelessness.

\section{Methods}

Details about the study design, study sample, and data collection were published elsewhere (Meerwijk, Chesla \& Weiss, 2014; Meerwijk, Ford \& Weiss, 2015). In short, we collected resting-state heart rate and EEG data in adults $(N=35)$ with a history of depression, who did not necessarily experience a depressive episode at the time of data collection. We 
107 intentionally enrolled participants who self-reported a current or past diagnosis of mood disorder,

108 as this was expected to result in a broad range of psychological pain in the sample. Participants

109 responded to flyers distributed to waiting rooms of a psychiatric hospital's outpatient department

110 and psychological services centers in the San Francisco Bay Area of California, and to online

111 advertisement on Craigslist from January to September 2012. A member of the research team

112 scheduled a time for study participation for respondents who met eligibility criteria and who

113 provided written informed consent. Neurophysiological data were recorded during 5-minute

114 sessions while participants were sitting upright and had been instructed to keep their eyes closed,

115 sit as still as possible, not focus on anything in particular and let their mind run free. The EEG

116 set up (Biosemi ActiveTwo, sample rate $1024 \mathrm{~Hz}$ ) involved 34 electrodes placed according to the

117 10/20 system. Horizontal and vertical electro-oculograms were recorded from the outer canthi of

118 the eyes and above and below the left eye. Two flat-type electrodes, one on the right collarbone

119 and one on the lower-left abdomen, were used to measure heart rate. Table 1 shows

120 sociodemographic characteristics of the sample. The Institutional Review Board at the University

121 of California, San Francisco, approved the study (IRB\# 11-07119).

\section{Psychological measures}

123

Psychological pain was assessed on the PS and OMMP and the order of administration was alternated to prevent testing effects. The PS has 13 items with higher scores reflecting greater psychological pain. Nine items (factor 1) are scored on a frequency scale ranging from never to always and four items reflecting pain intensity (factor 2) are scored on a symmetrical scale ranging from strongly disagree to strongly agree. The instrument is well validated in diverse populations, including outpatients with depression, male prison inmates, students at risk for suicide, and homeless men (Mills, Green \& Reddon, 2005; Patterson \& Holden, 2012; Troister \& Holden, 2012; Li et al., 2014; Xie et al., 2014). We found excellent internal consistency reliability with Cronbach's $\alpha=.92$. The OMMP assesses current psychological pain at the time of completing the questionnaire and worst-ever psychological pain, with higher total scores reflecting greater psychological pain. A 5-item response scale ranging between strongly disagree and strongly agree is used for all 44 items. The OMMP was shown to possess a high degree of validity in patients admitted for a suicide attempt, in university students and in the general population (Orbach et al., 2003a,b; Levi et al., 2008; Reisch et al., 2010; van Heeringen et al., 2010; Soumani et al., 2011; Nahaliel et al., 2014). We found excellent internal consistency reliability with Cronbach's $\alpha=.95$. Because the PS does not provide a comparison for worstever psychological pain, here we report on the current psychological pain dimension of the OMMP only. assess suicide ideation during the week that preceded the study. Suicidal desire was derived from 
143 BSS item 4, which indicates whether participants had an active suicidal desire. This item was

144 identified as having high discriminating power for assessing elevated suicide risk (De Beurs et

145 al., 2014).

146 Prior research has shown that psychological pain covaries with depression and

147 hopelessness. We included measures of depression and hopelessness to describe the sample and

148 to allow removal of the contribution of these constructs to the relationship with psychological

149 pain during statistical analysis. Participants completed the Beck Depression Inventory (BDI) II

150 (Beck, Steer \& Brown, 1996) and the Beck Hopelessness Scale ([BHS], Beck \& Steer, 1993).

$151 \quad$ Data processing and analysis

Heart rate data (lower left abdomen minus collarbone electrode, to maximize the signal and reduce signal noise) were visually inspected for artifacts and ectopic heart beats. Beat-tobeat intervals were determined using standard functions available from the BioSig 2.61 package for GNU Octave. We used fast Fourier transformation to determine low-frequency HRV and high-frequency HRV. Automated artifact detection was used to process frontal EEG data (F3/F4). Artifact-free data were divided into nonoverlapping 2 s epochs. Subsequently, EEG power was determined in standard EEG frequency bands (delta: $0.5-4 \mathrm{~Hz}$, theta: 4-8 Hz, alpha: 8-13 Hz, beta: 13-30 Hz, gamma: 30-100 Hz) and averaged per band across epochs. HRV and EEG power results were natural log transformed to obtain more normally distributed variables. Based on prior analysis regarding associations with psychological pain assessed on the OMMP and PS, we concentrated our analysis for hypothesis two on low-frequency HRV and right frontal EEG delta power (Meerwijk, Chesla \& Weiss, 2014; Meerwijk, Ford \& Weiss, 2015).

To test the moderating effect of recent suicidal desire, we created two groups, one group that indicated no suicidal desire and another group that indicated at least a weak suicidal desire (BSS item 4 score $>0$ ). Zero-order correlations were determined and simultaneous multiple regressions conducted to test the moderating effect of suicidal desire on the correlation between OMMP and PS (hypothesis one) and on the relationship between neurophysiological parameters and the PS score (hypothesis two). As hopelessness did not contribute significant variance to the regression models, we report models that control for level of depression only. $\mathrm{R}$ version 3.1.2 was used for all analyses, and statistical significance was assumed at $p<.05$.

\section{Results}

When comparing participants who reported a recent desire for suicide $(n=11)$ and participants with no desire for suicide or no recent desire for suicide $(n=24)$, the two groups did not differ with respect to sociodemographic characteristics (see Table 1). Table 2 shows clinical symptoms by suicidal desire. Based on their BDI scores, the majority of participants experienced moderate to severe depression. We found significant differences in depression and hopelessness, 
178 with more severe symptoms in participants with recent suicidal desire. They were also more

179 likely to report lifetime suicide attempts.

180 A strong positive correlation between PS and OMMP was observed in both groups (see

181 Table 3), although considerably stronger for the group with recent suicidal desire ( $r=.75, p<$

182.01 vs. $r=.50, p<.05)$. Strikingly, the correlation between PS factor 2 and the OMMP score in

183 the group with recent suicidal desire was very strong $(r=.90, p<.001)$, but only a statistical

184 trend existed for a correlation of medium strength in the group who reported no recent suicidal

185 desire $(r=.35, p=.09)$. The correlation between PS factor 1 and the OMMP score was strong

186 for both groups (recent suicidal desire, yes: $r=.64, p<.05$; no: $r=.51, p<.05$ ). As the total PS

187 score and factor scores are not independent and testing of multiple dependent correlations

188 inflates type I error (Curtin \& Schulz, 1998), we also evaluated the data using a Bonferroni

189 corrected significance level (.05 / 3 correlations). The correlations of the OMMP score with the

190 total PS and factor 2 score in the group with recent suicidal desire remained significant. When

191 we tested the association between PS factor 2 and OMMP in a linear model while controlling for

192 the level of depression, the overall model was significant $(F=9.08, d f=4,30, p<.0005)$ and a

193 significant interaction of Group $\times$ PS factor 2 was found $\left(\Delta R^{2}=.08, p<.05\right)$. However, this

194 interaction did not remain significant when using a Bonferroni correction for multiple tests.

195 Similar models with the total PS score and PS factor 1 did not show a significant interaction $\left(\Delta R^{2}\right.$

$196=.02, p=.17$ for Group $\times$ PS; $\Delta R^{2}=.00, p=.33$ for Group $\times$ PS factor 1). Effect sizes indicate

197 that hypothesis 1 was supported primarily for factor 2 of the Psychache Scale. Small sample size

198 precluded reaching significance levels in some of these analyses.

199

Findings with respect to hypothesis two are presented in Tables 4 and 5. Table 4 shows

200 regression models of the PS score and PS factor scores on right frontal delta power, while

201 controlling for depression and recent suicidal desire. A significant interaction of Group $\times$ delta

202 power was observed $\left(\Delta R^{2}=.07, p<.01\right)$ with opposite associations between the PS score and

203 delta power in the groups with $(\beta=-.37, p=.20)$ and without $(\beta=.33, p<.01)$ recent suicidal

204 desire. Similar Group $\times$ delta power interaction effects were observed when the PS factor scores

205 were regressed on delta power, with the interaction term accounting for $\Delta R^{2}=.05(p<.05)$ in the

206 model of PS factor 1 and $\Delta R^{2}=.08(p<.05)$ in the model of PS factor 2 . Table 4 also shows that

207 no interaction effect was observed when the OMMP score was regressed on right frontal delta

208 power.

209

210

Table 5 shows regression models of the PS score and PS factor scores on low-

211

frequency HRV, while controlling for depression and recent suicidal desire. All overall models

211 were significant, but none of the models showed a significant interaction of Group $\times$ low-

212 frequency HRV. Post-hoc power analysis indicated a high risk for type II error $(\beta=.64)$, given

213 the size of the interaction effect for the model that included the PS total score (partial $\eta^{2}=.073$ ).

214 While statistically nonsignificant, all three models suggested opposite directions for the 
215 association between the psychological pain score and low-frequency HRV in the groups with and 216 without recent suicidal desire (respectively, $\beta=-.22, p=.46$ and $\beta=.30, p<.05$ for the model

217 that included the PS total score). No indication for an interaction effect was observed when the

218 OMMP score was regressed on low-frequency HRV (see Table 5).

219

220

221

222

223

224

225

226

227

228

229

230

231

232

233

234

235

236

237

238

239

240

241

242

243

244

245

246

247

248

249

250

The difference in effect sizes for regression models of right frontal delta power and low-frequency HRV prompted us to explore the association between low-frequency HRV and right frontal delta power. We regressed low-frequency HRV on right frontal delta power while controlling for depression, recent suicidal desire, and age, as age covaried with HRV. The overall model was significant $(F=3.55, d f=3,31, p<.05)$, and right frontal delta power was positively associated with low-frequency $\operatorname{HRV}(\beta=.36, p<.05)$, confirming that the effect of recent suicidal desire on the association between low-frequency HRV and the PS score may have been nonsignificant due to a lack of power. There was no evidence of opposite associations between right-frontal delta power and low-frequency HRV for the groups with and without recent suicidal desire.

\section{Discussion}

The present study was a secondary analysis to test the moderating effect of recent suicidal desire on 1) correlations between the OMMP and the PS, and 2) on relationships between psychological pain and resting-state neurophysiological parameters. We found corroborating evidence for our hypothesis that the correlation between psychological pain as assessed on the OMMP and the PS depends on recent suicidal desire. This is especially clear for the pain intensity factor of the PS, which revealed a very strong positive association in participants with suicidal desire within the last week, while controlling for depression. In participants without recent suicidal desire the association between PS pain intensity (factor two) and OMMP was also positive, but of moderate strength and not statistically significant. For PS pain frequency (factor 1) and the total PS score, the differences were not as pronounced, but they too indicated considerably stronger associations for participants with recent suicidal desire.

Analysis of low-frequency HRV and right frontal EEG delta power provided evidence that recent desire for suicide moderates the relationship between the PS score and neurophysiological parameters. This was evident for EEG delta power, essentially confirming our second hypothesis, and to a lesser extent for low-frequency HRV. The significant positive correlation between low-frequency HRV and EEG delta power, while controlling for age and level of depressive symptoms, strengthens our interpretation that the difference in association between PS and low-frequency HRV in participants with and without recent suicidal desire would likely be statistically significant in a larger sample. Among participants with recent suicidal desire, EEG delta power and low-frequency HRV were both lower when individuals reported greater psychological pain on the PS. The direction and strength of these associations 
251 resemble findings in the complete sample for associations between OMMP and low-frequency

252 HRV and EEG delta power, reported elsewhere (Meerwijk, Chesla \& Weiss, 2014; Meerwijk,

253 Ford \& Weiss, 2015). In that study, decreased low-frequency HRV and EEG delta power were

254 interpreted as indicators of less effective emotion regulation, including increased rumination and

255 an inability to reappraise the causes and consequences of psychological pain. There is evidence

256 that variability in resting-state heart rate and EEG reflects emotion regulation processes

257 (Beauchaine, 2001; Thayer et al., 2009; Bornas et al., 2012; Knyazev, 2012). Rumination and

258

259

260

261

262

263

264

265

266

267

268

269

270

271

272

273

274

275

276

277

278

279

280

281

282

283

284

285

286

287 cognitive reappraisal are recognized examples of emotion regulation strategies (Aldao, NolenHoeksema \& Schweizer, 2010). Interestingly, we found opposite results among participants who reported no suicidal desire during the past week. In this group, low-frequency HRV and EEG delta power were higher when individuals reported greater psychological pain on the PS. We speculate that participants without recent suicidal desire may have had better emotion regulation skills. The majority of participants without recent desire for suicide (87.5\%) had never attempted suicide, whereas a third of participants with recent suicidal desire had attempted suicide more than once. Research has shown that poor emotion regulation is a distinguishing factor between multiple attempters and individuals who attempted suicide once (Reynolds \& Eaton, 1986; Choi et al., 2013). Moreover, rumination in psychiatric inpatients was positively associated with suicide ideation, and cognitive reappraisal was significantly higher in psychiatric inpatients who reported no suicide ideation (Morrison \& O'Connor, 2008; Forkmann et al., 2014). However, the study by Forkmann et al. did not find differences based on suicidal desire.

The results corroborate our assumption that the PS and OMMP perform more similarly in the presence of recent or current psychological pain that is expected in individuals with recent suicidal desire. In contrast, the scales appear to diverge in individuals without recent suicidal desire. In this latter group, respondents may have reported on current pain in one measure (the OMMP) and on psychological pain over time or at a highly significant previous time in the other measure (PS). We postulate that a change in the instructions for the PS could improve concurrent validity of the two scales. The OMMP specifically instructs respondents to consider current psychological pain, whereas the PS leaves the time frame undefined. Although this may not be an issue in a population that is likely to experience psychological pain at the time of completing the PS, it does become relevant when study participants are not homogeneous with respect to when they experienced psychological pain, as was the case in our study. Given the robust relationship between suicide and psychological pain, it may be that participants who did not report a recent desire for suicide did not experience significant psychological pain recently either. We would expect the OMMP to capture this distinction, resulting in lower scores. Due to its more general instructions, these same participants may have completed the PS while reflecting on psychological pain they experienced at some undefined time in the past, resulting in higher scores than their current level of psychological pain warranted. It is likely that including a 
288

289

290

291

292

293

294

295

296

297

298

299

300

301

302

303

304

305

306

307

308

309

310

311

312

313

314

315

316

317

318

319

320

321

322

323

324

time frame in the measure's instructions, for example psychological pain during the past week, would address the issue. This would emphasize that the PS is about recent psychological pain and not about psychological pain experienced during some distant past. A similar instruction is used for the BSS, which assesses suicide ideation during the past week. If recency of psychological pain affects how individuals complete the PS, making the instrument's instructions more specific will enhance the PS's clinical applicability and facilitate comparison of PS scores between populations and comparison with correlates of psychological pain.

Alternatively, our results could reflect a genuine difference in sensitivity for suicidal desire in the items of the PS and OMMP, for which support can be found in the origin of the two scales. The PS items were derived from Shneidman's theory of suicide (Holden et al., 2001), whereas the OMMP items describe different aspects of psychological pain and were based on narratives and interviews with individuals familiar with psychological pain due to aversive life events or personal issues (Orbach et al., 2003a). As such, it is not unreasonable to assume that the PS may be more sensitive to suicidal thoughts and behaviors. It is noteworthy that the PS score in the group with recent suicidal desire is significantly higher than in the group without recent suicidal desire, whereas the OMMP score did not differ significantly between the two groups. This could suggest that the PS and OMMP are sensitive to different underlying processes. Another obvious difference between the two scales is that the PS assesses both intensity and frequency of the pain, whereas the OMMP assesses intensity only. The impact of these different characteristics on assessment of psychological pain is not known.

Some limitations of this study should be addressed. The available sample size was small, especially for participants who reported a recent desire for suicide. Therefore, insufficient power may explain why some of our subgroup analyses produced nonsignificant results or reached trend-level significance only. However, despite a small sample size, we found significant interaction effects for suicidal desire, which suggests that the findings are unlikely to be a result of chance alone. Our sample was not homogeneous with respect to the participants' diagnosis of depression. To compensate for the differences in depression we controlled our analyses for the level of depressive symptoms. Participants knew they enrolled in a study about psychological pain. Although we did not disclose our specific hypotheses, participants' opinions about the purpose of the study may have affected how they completed the self-report instruments. However, our significant results involving neurophysiological parameters, which are objective by nature, suggest that findings are not likely the result of participant bias.

Our results suggest that recent suicidal desire moderates psychological pain assessed on the PS, whereas no such moderation existed for OMMP scores. We conclude that this moderating effect is not a genuine difference in the overall constructs being measured or sensitivity of the PS, but may be an artifact of the instrument's instructions with regard to the time frame when psychological pain was experienced. Our recommendation for changes to the 
325 instructions for completion of the PS could enhance its clinical applicability. Additional research

326 is necessary to assess the effect of such a modification on concurrent validity of the PS and if

327 such a modification leads to significant associations with resting-state neurophysiological

328 parameters similar to those found previously for the OMMP. 
331

332

333

334

335

336

337

338

339

340

341

342

343

344

345

346

347

348

349

350

351

352

353

354

355

356

357

358

359

360

361

362

363

364

365

Aldao A., Nolen-Hoeksema S., Schweizer S. 2010. Emotion-regulation strategies across psychopathology: A meta-analytic review. Clinical psychology review 30:217-37.

Beauchaine T. 2001. Vagal tone, development, and Gray's motivational theory: toward an integrated model of autonomic nervous system functioning in psychopathology. Development and psychopathology 13:183-214.

Beck AT., Brown GK., Steer RA. 1997. Psychometric characteristics of the Scale for Suicide Ideation with psychiatric outpatients. Behaviour Research and Therapy 35:1039-1046.

Beck AT., Steer RA. 1993. Beck Hopelessness Scale Manual. San Antonio, TX: Pearson.

Beck AT., Steer RA., Brown GK. 1996. BDI-II Manual. San Antonio, TX: Pearson.

De Beurs DP., de Vries AL., de Groot MH., de Keijser J., Kerkhof AJ. 2014. Applying computer adaptive testing to optimize online assessment of suicidal behavior: a simulation study. Journal of medical Internet research 16:e207.

Bornas X., Tortella-Feliu M., Balle M., Llabrés J., Llabres J. 2012. Self-focused cognitive emotion regulation style as associated with widespread diminished EEG fractal dimension. International Journal of Psychology 48:695-703.

Cáceda R., Durand D., Cortes E., Prendes-Alvarez S., Moskovciak T., Harvey PD., Nemeroff CB. 2014. Impulsive Choice and Psychological Pain in Acutely Suicidal Depressed Patients. Psychosomatic Medicine 76:445-451.

Chang H-A., Chang C-C., Chen C-L., Kuo TBJ., Lu R-B., Huang S-Y. 2012. Major depression is associated with cardiac autonomic dysregulation. Acta Neuropsychiatrica 24:318-327.

Choi KH., Wang S-M., Yeon B., Suh S-Y., Oh Y., Lee H-K., Kweon Y-S., Lee CT., Lee K-U. 2013. Risk and protective factors predicting multiple suicide attempts. Psychiatry research 210:957-61.

Curtin F., Schulz P. 1998. Multiple correlations and Bonferroni's correction. Biological psychiatry 44:775-7.

DeLisle MM., Holden RR. 2009. Differentiating between depression, hopelessness, and psychache in university undergraduates. Measurement and Evaluation in Counseling and Development 42:46-63.

Flamenbaum R., Holden RR. 2007. Psychache as a mediator in the relationship between perfectionism and suicidality. Journal of Counseling Psychology 54:51-61.

Forkmann T., Scherer A., Böcker M., Pawelzik M., Gauggel S., Glaesmer H. 2014. The relation of cognitive reappraisal and expressive suppression to suicidal ideation and suicidal desire. Suicide \& life-threatening behavior 44:524-36.

van Heeringen K., Van den Abbeele D., Vervaet M., Soenen L., Audenaert K. 2010. The functional neuroanatomy of mental pain in depression. Psychiatry Research 181:141-144. 
366

367

368

369

370

371

372

373

374

375

376

377

378

379

380

381

382

383

384

385

386

387

388

389

390

391

392

393

394

395

396

397

398

399

400

401

402

403

404

Holden RR., Mehta K., Cunningham EJ., McLeod LD. 2001. Development and preliminary validation of a scale of psychache. Canadian Journal of Behavioural Science 33:224-232.

Hunter AM., Leuchter AF., Cook IA., Abrams M. 2010. Brain functional changes (QEEG cordance) and worsening suicidal ideation and mood symptoms during antidepressant treatment. Acta Psychiatrica Scandinavica 122:461-9.

Iosifescu D V., Greenwald S., Devlin P., Perlis RH., Denninger JW., Alpert JE., Fava M. 2008. Pretreatment frontal EEG and changes in suicidal ideation during SSRI treatment in major depressive disorder. Acta Psychiatrica Scandinavica 117:271-276.

Knyazev GG. 2012. EEG delta oscillations as a correlate of basic homeostatic and motivational processes. Neuroscience and Biobehavioral Reviews 36:677-695.

Leenaars AA., Lester D. 2004. A Note on Shneidman's Psychological Pain Assessment Scale. Omega: Journal of Death and Dying 50:2004-2005.

Lester D. 2000. Psychache, depression, and personality. Psychological Reports 87:940.

Levi Y., Horesh N., Fischel T., Treves I., Or E., Apter A. 2008. Mental pain and its communication in medically serious suicide attempts: an "impossible situation." Journal of Affective Disorders 111:244-250.

Li H., Xie W., Luo X., Fu R., Shi C., Ying X., Wang N., Yin Q., Wang X. 2014. Clarifying the role of psychological pain in the risks of suicidal ideation and suicidal acts among patients with major depressive episodes. Suicide \& life-threatening behavior 44:78-88.

Mee S., Bunney BG., Bunney WE., Hetrick W., Potkin SG., Reist C. 2011. Assessment of psychological pain in major depressive episodes. Journal of Psychiatric Research 45:15041510 .

Meerwijk EL., Chesla CA., Weiss SJ. 2014. Psychological Pain and Reduced Resting-State Heart Rate Variability in Adults with a History of Depression. Psychophysiology 51:24756.

Meerwijk EL., Ford JM., Weiss SJ. 2015. Resting-state EEG delta power is associated with psychological pain in adults with a history of depression. Biological psychology 105:106114.

Meerwijk EL., Weiss SJ. 2011. Toward a unifying definition of psychological pain. Journal of Loss \& Trauma 16:402-412.

Mills JF., Green K., Reddon JR. 2005. An evaluation of the psychache scale on an offender population. Suicide and Life-Threatening Behavior 35:570-580.

Morrison R., O'Connor RC. 2008. A systematic review of the relationship between rumination and suicidality. Suicide \& life-threatening behavior 38:523-38.

Nahaliel S., Sommerfeld E., Orbach I., Weller A., Apter A., Zalsman G. 2014. Mental pain as a mediator of suicidal tendency: A path analysis. Comprehensive Psychiatry 55:944-951.

Olié E., Guillaume S., Jaussent I., Courtet P., Jollant F. 2010. Higher psychological pain during a major depressive episode may be a factor of vulnerability to suicidal ideation and act. Journal of Affective Disorders 120:226-230. 
405

406

407

408

409

410

411

412

413

414

415

416

417

418

419

420

421

422

423

424

425

426

427

428

429

430

431

432

433

434

435

436

437

438

439

440

441

442

443

Orbach I., Mikulincer M., Sirota P., Gilboa-Schechtman E. 2003a. Mental pain: A multidimensional operationalization and definition. Suicide and Life-Threatening Behavior $33: 219-230$.

Orbach I., Mikulincer M., Gilboa-Schechtman E., Sirota P. 2003b. Mental pain and its relationship to suicidality and life meaning. Suicide and Life-Threatening Behavior 33:231241.

Patterson AA., Holden RR. 2012. Psychache and suicide ideation among men who are homeless: a test of Shneidman's model. Suicide and Life-Threatening Behavior 42:147-156.

Pereira EJ., Kroner DG., Holden RR., Flamenbaum R. 2010. Testing Shneidman's model of suicidality in incarcerated offenders and in undergraduates. Personality and Individual Differences 49:912-917.

Pompili M., Lester D., Leenaars AA., Tatarelli R., Girardi P. 2008. Psychache and suicide: A preliminary investigation. Suicide and Life-Threatening Behavior 38:116-121.

Pompili M., Innamorati M., Szanto K., Di Vittorio C., Conwell Y., Lester D., Tatarelli R., Girardi P., Amore M. 2011. Life events as precipitants of suicide attempts among first-time suicide attempters, repeaters, and non-attempters. Psychiatry Research 186:300-5.

Reisch T., Seifritz E., Esposito F., Wiest R., Valach L., Michel K. 2010. An fMRI study on mental pain and suicidal behavior. Journal of Affective Disorders 126:321-325.

Reynolds P., Eaton P. 1986. Multiple attempters of suicide presenting at an emergency department. Canadian journal of psychiatry 31:328-30.

Shneidman ES. 1999. The psychological pain assessment scale. Suicide and Life-Threatening Behavior 29:287-294.

Song BA., Yoo SY., Kang HY., Byeon SH., Shin SH., Hwang EJ., Lee SH. 2011. PostTraumatic Stress Disorder, Depression, and Heart-Rate Variability among North Korean Defectors. Psychiatry Investigation 8:297-304.

Soumani A., Damigos D., Oulis P., Masdrakis V., Ploumpidis D., Mavreas V., Konstantakopoulos G. 2011. Mental pain and suicide risk: Application of the greek version of the Mental Pain and the Tolerance of Mental Pain scale. Psychiatriki 22:330-340.

Thayer JF., Hansen AL., Saus-Rose E., Johnsen BH. 2009. Heart rate variability, prefrontal neural function, and cognitive performance: the neurovisceral integration perspective on self-regulation, adaptation, and health. Annals of behavioral medicine : a publication of the Society of Behavioral Medicine 37:141-53.

Tossani E. 2013. The concept of mental pain. Psychotherapy and Psychosomatics 82:67-73.

Troister T., Davis MP., Lowndes A., Holden RR. 2013. A five-month longitudinal study of psychache and suicide ideation: replication in general and high-risk university students. Suicide \& life-threatening behavior 43:611-20.

Troister T., D'Agata MT., Holden RR. 2015. Suicide Risk Screening: Comparing the Beck Depression Inventory-II, Beck Hopelessness Scale, and Psychache Scale in Undergraduates. Psychological assessment. 
444 Troister T., Holden RR. 2010. Comparing psychache, depression, and hopelessness in their

445

446

447 448

449 450 451

452 453

454

455

456 associations with suicidality: A test of Shneidman's theory of suicide. Personality and Individual Differences 49:689-693.

Troister T., Holden RR. 2012. A two-year prospective study of psychache and its relationship to suicidality among high-risk undergraduates. Journal of Clinical Psychology 68:1019-1027.

Troister T., Holden RR. 2013. Factorial Differentiation Among Depression, Hopelessness, and Psychache in Statistically Predicting Suicidality. Measurement and Evaluation in Counseling and Development 46:50-63.

Xie W., Li H., Luo X., Fu R., Ying X., Wang N., Yin Q., Zou Y., Cui Y., Wang X., Shi C. 2014. Anhedonia and pain avoidance in the suicidal mind: behavioral evidence for motivational manifestations of suicidal ideation in patients with major depressive disorder. Journal of clinical psychology 70:681-92. 


\section{Table $\mathbf{1}$ (on next page)}

Sociodemographic characteristics by recent suicidal desire.

${ }^{a}$ all $\chi^{2}$ tests included cells with expected counts less than $5,{ }^{b}$ Other included native Hawaiian, Pacific Islander, native American, and Alaska native. 
1 Table 1 Sociodemographic Characteristics by Recent Suicidal Desire.

\begin{tabular}{|c|c|c|c|c|}
\hline & \multicolumn{2}{|c|}{ Recent Suicidal Desire } & \multirow[b]{2}{*}{$\begin{array}{c}\text { Total } \\
(N=35)\end{array}$} & \\
\hline & $\begin{array}{c}\text { No } \\
(n=24)\end{array}$ & $\begin{array}{c}\text { Yes } \\
(n=11)\end{array}$ & & \\
\hline Mean age in years $(S D)$ & $35.42(11.41)$ & $34.18(13.27)$ & $34.91(11.60)$ & $t=0.28$ \\
\hline Gender & & & & $\chi^{2}=0.18^{\mathrm{a}}$ \\
\hline Men & 5 & 3 & 8 & \\
\hline Women & 19 & 8 & 17 & \\
\hline Ethnicity & & & & $\chi^{2}=1.32$ \\
\hline White & 13 & 4 & 17 & \\
\hline African American & 2 & 1 & 3 & \\
\hline Hispanic & 2 & 2 & 4 & \\
\hline Asian & 3 & 2 & 5 & \\
\hline Other or mixed ${ }^{b}$ & 4 & 2 & 6 & \\
\hline Marital status & & & & $\chi^{2}=1.25$ \\
\hline Single & 21 & 10 & 31 & \\
\hline Married / in a relationship & 3 & 1 & 4 & \\
\hline Highest education & & & & $\chi^{2}=0.89$ \\
\hline High school & 3 & 2 & 5 & \\
\hline Some college & 6 & 4 & 10 & \\
\hline College graduate & 9 & 3 & 12 & \\
\hline Graduate/professional school & 6 & 2 & 8 & \\
\hline Employment status & & & & $\chi^{2}=1.66$ \\
\hline Unemployed & 11 & 3 & 14 & \\
\hline Occasionally employed & 6 & 5 & 11 & \\
\hline Regularly employed & 7 & 3 & 10 & \\
\hline
\end{tabular}

${ }^{\mathrm{a}}$ all $\chi^{2}$ tests included cells with expected counts less than $5,{ }^{\mathrm{b}}$ Other included native Hawaiian, Pacific Islander, native American, and Alaska native.

2

3

4 


\section{Table 2 (on next page)}

Clinical characteristics (mean or frequency) by recent suicidal desire.

Note. PS: psychache scale, OMMP: Orbach \& Mikulincer current mental pain questionnaire, BDI: Beck depression inventory, BHS: Beck hopelessness scale, $\mathrm{D}_{\mathrm{x}}$ : diagnosis, NOS: not otherwise specified. ${ }^{*} p<.05,{ }^{*} p<.01$. 
1 Table 2 Clinical Characteristics (Mean or Frequency) by Recent Suicidal Desire.

\begin{tabular}{|c|c|c|c|c|}
\hline \multicolumn{5}{|c|}{ Recent Suicidal Desire } \\
\hline & $\begin{array}{c}\text { No } \\
(n=24)\end{array}$ & $\begin{array}{c}\text { Yes } \\
(n=11)\end{array}$ & $\begin{array}{c}\text { Total } \\
(N=35)\end{array}$ & \\
\hline $\mathrm{PS}(S D)$ & $37.4(9.5)$ & $47.6(8.9)$ & $40.6(10.4) t$ & $t=-2.98^{* *}$ \\
\hline OMMP $(S D)$ & $117.2(26.8)$ & $132.6(28.0)$ & $122.0(27.7) t$ & $t=-1.57$ \\
\hline $\mathrm{BDI}(S D)$ & $24.4(10.3)$ & $33.4(9.8)$ & $27.2(10.8) t$ & $t=-2.43^{*}$ \\
\hline $\mathrm{BHS}(S D)$ & $10.2(5.0)$ & $15.1(4.3)$ & $11.7(5.3) \quad t$ & $t=-2.81^{* *}$ \\
\hline Years since $\mathrm{D}_{\mathrm{x}}(S D)$ & $7.2(6.6)$ & $5.9(5.4)$ & $6.9(6.3)$ & $t=-0.52$ \\
\hline Diagnosis & & & & $\chi^{2}=6.40^{*}$ \\
\hline Major depressive disorder & 15 & 2 & 17 & \\
\hline Dysthymic disorder & 1 & 2 & 3 & \\
\hline Depression NOS & 8 & 7 & 15 & \\
\hline Currently on antidepressants & & & & $\chi^{2}=0.50$ \\
\hline yes & 14 & 5 & 19 & \\
\hline no & 10 & 6 & 16 & \\
\hline Suicide attempts & & & & $\chi^{2}=7.91^{*}$ \\
\hline Never & 21 & 5 & 26 & \\
\hline Once & 2 & 2 & 4 & \\
\hline More than once & 1 & 4 & 5 & \\
\hline
\end{tabular}

Note. PS: psychache scale, OMMP: Orbach \& Mikulincer current mental pain questionnaire, BDI: Beck depression inventory, BHS: Beck hopelessness scale, $\mathrm{D}_{\mathrm{x}}$ : diagnosis, NOS: not otherwise specified.

${ }^{*} p<.05,{ }^{* *} p<.01$. 


\section{Table 3 (on next page)}

Zero-order correlations between clinical symptoms by recent suicidal desire.

Note. Group with recent suicidal desire $(n=11)$ shown below the diagonal and without recent suicidal desire $(n=24)$ above the diagonal. PS: psychache scale, OMMP: Orbach \& Mikulincer current mental pain questionnaire, BDI: Beck depression inventory, BHS: Beck hopelessness scale, BSS: Beck Scale for Suicide ideation minus item 4 to maintain independence. ${ }^{\text {a }}$ Spearman instead of Pearson correlations because of nonnormality; ${ }^{b}$ PS factor 1 contains nine frequency items, PS factor 2 contains four intensity items. ${ }^{* * *} p<.001$, ${ }^{* *} p<.01,{ }^{*} p<.05 .{ }^{\dagger} p<.10$ 
1 Table 3 Zero-Order Correlations Between Clinical Symptoms by Recent Suicidal Desire.

2

\begin{tabular}{|c|c|c|c|c|c|c|c|}
\hline & 1 & 2 & 3 & 4 & 5 & 6 & $7^{\mathrm{a}}$ \\
\hline 1. PS & - & $.97^{* * *}$ & $.80^{* * *}$ & $.50^{*}$ & $.78^{* * *}$ & $.50^{*}$ & .21 \\
\hline 2. PS factor $1^{\mathrm{b}}$ & $.98^{* * *}$ & - & $.64^{* * *}$ & $.51^{*}$ & $.74^{* * *}$ & $.47^{*}$ & .16 \\
\hline 3. $\mathrm{PS}$ factor $2^{\mathrm{b}}$ & $.82^{* *}$ & $.70^{*}$ & - & $.35^{\dagger}$ & $.68^{* * *}$ & $.44^{*}$ & $.42^{*}$ \\
\hline 4. OMMP & $.75^{* *}$ & $.64^{*}$ & $.90^{* * *}$ & - & $.66^{* * *}$ & $.60^{* *}$ & .27 \\
\hline 5. BDI & $.57^{\dagger}$ & $.53^{\dagger}$ & $.57^{\dagger}$ & $.58^{\dagger}$ & - & $.54^{* *}$ & $.58^{* *}$ \\
\hline 6. BHS & .33 & .23 & $.55^{\dagger}$ & .52 & $.64^{*}$ & - & $.51^{*}$ \\
\hline 7. $\mathrm{BSS}^{\mathrm{a}}$ & .07 & -.07 & .51 & 46 & $.58^{\dagger}$ & $.57^{\dagger}$ & - \\
\hline
\end{tabular}

Note. Group with recent suicidal desire $(n=11)$ shown below the diagonal and without recent suicidal desire $(n=24)$ above the diagonal. PS: psychache scale, OMMP: Orbach \& Mikulincer current mental pain questionnaire, BDI: Beck depression inventory, BHS: Beck hopelessness scale, BSS: Beck Scale for Suicide ideation minus item 4 to maintain independence.

${ }^{a}$ Spearman instead of Pearson correlations because of nonnormality; ${ }^{b}$ PS factor 1 contains nine frequency items, PS factor 2 contains four intensity items.

${ }^{* * *} p<.001,{ }^{* *} p<.01,{ }^{*} p<.05 .,{ }^{\dagger} p<.10$ 


\section{Table 4 (on next page)}

Simultaneous multiple regression models of psychological pain on right frontal EEG delta power controlling for level of depression and recent suicidal desire.

Note. PS: psychache scale, BDI: Beck depression inventory, F4 delta: grand mean centered right midfrontal EEG delta power in $\ln \left(\mu \mathrm{V}^{2}\right)$, SD: recent suicidal desire (yes/no), OMMP:

Orbach \& Mikulincer current mental pain questionnaire. ${ }^{\text {a }}$ PS factor 1 contains nine frequency items, PS factor 2 contains four intensity items. ${ }^{* * *} p<.001,{ }^{* *} p<.01,{ }^{*} p<.05,{ }^{\dagger}<.10$. 


\begin{tabular}{|c|c|c|c|c|c|}
\hline \multirow[t]{2}{*}{ Table 4} & \multirow[b]{2}{*}{$F$} & \multirow[b]{2}{*}{$R^{2}$} & \multirow[b]{2}{*}{$b$} & \multirow[b]{2}{*}{$S E(b)$} & \multirow[t]{2}{*}{$\begin{array}{l}\text { Right Fror } \\
\text { dicidal }\end{array}$} \\
\hline & & & & & \\
\hline PS & $19.13^{* * *}$ & $68.1 \%$ & & & \\
\hline BDI & & & 0.69 & 0.10 & $.72^{* * *}$ \\
\hline F4 delta & & & 5.59 & 2.18 & $.28^{*}$ \\
\hline $\mathrm{SD}$ & & & 4.38 & 2.32 & $.20^{\dagger}$ \\
\hline $\mathrm{SD} \times \mathrm{F} 4$ delta & & & -14.04 & 4.98 & $-.31^{* *}$ \\
\hline PS factor $1^{\mathrm{a}}$ & $13.61^{* * *}$ & $59.7 \%$ & & & \\
\hline BDI & & & 0.06 & 0.01 & $.68^{* * *}$ \\
\hline F4 delta & & & 0.38 & 0.21 & $.22^{\dagger}$ \\
\hline $\mathrm{SD}$ & & & 0.41 & 0.23 & $.21^{\dagger}$ \\
\hline $\mathrm{SD} \times \mathrm{F} 4$ delta & & & -1.11 & 0.49 & $-.28^{*}$ \\
\hline PS factor $2^{\mathrm{a}}$ & $13.39^{* * *}$ & $59.3 \%$ & & & \\
\hline BDI & & & 0.04 & 0.01 & $.67^{* * *}$ \\
\hline F4 delta & & & 0.53 & 0.17 & $.38^{* *}$ \\
\hline $\mathrm{SD}$ & & & 0.18 & 0.18 & .12 \\
\hline $\mathrm{SD} \times \mathrm{F} 4$ delta & & & -1.01 & 0.39 & $-.32^{*}$ \\
\hline OMMP & $8.25^{* * *}$ & $46.0 \%$ & & & \\
\hline BDI & & & 1.77 & 0.35 & $.69^{* * *}$ \\
\hline F4 delta & & & -11.61 & 7.57 & -.22 \\
\hline $\mathrm{SD}$ & & & 1.73 & 8.08 & .03 \\
\hline $\mathrm{SD} \times \mathrm{F} 4$ delta & & & -14.34 & 17.31 & -.12 \\
\hline
\end{tabular}

Note. PS: psychache scale, BDI: Beck depression inventory, F4 delta: grand mean centered right midfrontal EEG delta power in $\ln \left(\mu \mathrm{V}^{2}\right)$, SD: recent suicidal desire (yes/no), OMMP: Orbach \& Mikulincer current mental pain questionnaire.

a PS factor 1 contains nine frequency items, PS factor 2 contains four intensity items. 


\section{Table 5 (on next page)}

Simultaneous multiple regression models of psychological pain on low-frequency heart rate variability controlling for level of depression and recent suicidal desire.

Note. PS: psychache scale, BDI: Beck depression inventory, LF HRV: grand mean centered low-frequency heart rate variability in $\ln \left(\mathrm{ms}^{2}\right)$, SD: recent suicidal desire (yes/no), OMMP:

Orbach \& Mikulincer current mental pain. ${ }^{a}$ PS factor 1 contains nine frequency items, PS factor 2 contains four intensity items. ${ }^{* * *} p<.001,{ }^{*} p<.05,{ }^{\dagger}<.10$. 
1 Table 5 Simultaneous Multiple Regression Models of Psychological Pain on Low-

2

3

4
Frequency Heart Rate Variability Controlling for Level of Depression and Recent Suicidal Desire.

\begin{tabular}{|c|c|c|c|c|c|}
\hline & $F$ & $R^{2}$ & $b$ & $S E(b)$ & $\beta$ \\
\hline PS & $15.59^{* * *}$ & $63.2 \%$ & & & \\
\hline BDI & & & 0.70 & 0.11 & $.73^{* * *}$ \\
\hline LF HRV & & & 2.57 & 1.28 & $.24^{\dagger}$ \\
\hline SD & & & 4.43 & 2.85 & .20 \\
\hline $\mathrm{SD} \times \mathrm{LF} \mathrm{HRV}$ & & & -5.24 & 3.40 & -.20 \\
\hline PS factor $1^{\mathrm{a}}$ & $13.22^{* * *}$ & $59.0 \%$ & & & \\
\hline BDI & & & 0.06 & 0.01 & $.70^{* * *}$ \\
\hline LF HRV & & & 0.26 & 0.12 & $.28^{*}$ \\
\hline $\mathrm{SD}$ & & & 0.38 & 0.26 & .20 \\
\hline $\mathrm{SD} \times \mathrm{LF} \mathrm{HRV}$ & & & -0.44 & 0.31 & -.19 \\
\hline PS factor $2^{\mathrm{a}}$ & $8.17^{* * *}$ & $45.8 \%$ & & & \\
\hline BDI & & & 0.04 & 0.01 & $.66^{* * *}$ \\
\hline LF HRV & & & 0.06 & 0.11 & .08 \\
\hline $\mathrm{SD}$ & & & 0.26 & 0.24 & .17 \\
\hline $\mathrm{SD} \times \mathrm{LF} \mathrm{HRV}$ & & & -0.32 & 0.29 & -.17 \\
\hline OMMP & $10.59^{* * *}$ & $53.0 \%$ & & & \\
\hline BDI & & & 1.50 & 0.33 & $.59^{* * *}$ \\
\hline LF HRV & & & -9.28 & 3.85 & $-.32^{*}$ \\
\hline $\mathrm{SD}$ & & & 11.99 & 8.61 & .20 \\
\hline $\mathrm{SD} \times \mathrm{LF} \mathrm{HRV}$ & & & -11.36 & 10.26 & -.16 \\
\hline
\end{tabular}

Note. PS: psychache scale, BDI: Beck depression inventory, LF HRV: grand mean centered low-frequency heart rate variability in $\ln \left(\mathrm{ms}^{2}\right)$, SD: recent suicidal desire (yes/no), OMMP: Orbach \& Mikulincer current mental pain.

a PS factor 1 contains nine frequency items, PS factor 2 contains four intensity items.

${ }^{* * *} p<.001,{ }^{*} p<.05, \dagger<.10$. 